\title{
The use of CorMatrix extracellular matrix for aortic root enlargement
}

Derek R Brinster ${ }^{1,2^{*}}$ and Jay A Patel ${ }^{1,2}$

\begin{abstract}
This manuscript presents a published record of the use and efficacy of the extracellular matrix CorMatrix and why it may be superior to other materials used for aortic root enlargement. The potential benefits of an extracellular matrix are the natural ingrowth and development of native arterial cells and structure.
\end{abstract}

Keywords: Aorta, Aortic valve replacement, Aortic root enlargement, CorMatrix, Extracellular matrix

\section{Background}

Aortic root enlargement (ARE) is a surgical procedure used to widen the annulus of the aortic root to facilitate aortic valve replacement (AVR). ARE is sometimes necessary to prevent patient-prosthesis mismatch, which occurs when the area of the prosthesis is insufficient relative to the size of the patient, and is associated with higher rates mortality [1]. Both the orifice area of the prosthesis and the resulting pressure gradient across the aortic valve are important determinants to successful AVR. Procedures used to enlarge the aortic root include the Nicks' Procedure which is the most common, Manougian Technique, Seybold-Epting Technique, modified Bentall Procedure, and aortoventriculoplasty. During AVR, an effective means towards repair and augmentation of the aortic root is necessary. Materials currently used for reconstruction are Dacron (cloth), polytetrafluroethlyene (polymer), and bovine/patient derived pericardium [2]. Significant drawbacks to these materials include difficulty in resorption and difficulty in promoting full functioning of the reconstructed area [2]. An important alternative to these methods is the CorMatrix ECM, an extracellular matrix (ECM) derived from porcine small intestinal submucosa [3], which includes a matrix of structural proteins normally found outside cells that provide tissue support.

\footnotetext{
* Correspondence: derekbrinster@yahoo.com
'Department of Cardiothoracic Surgery, Virginia Commonwealth University,

* Correspondence: derekbrinster@yahoo.com
${ }^{1}$ Department of Cardiothoracic Surgery, Virginia Commonwealth University, Richmond, VA, USA

${ }^{2}$ Divisions of Cardiothoracic and Vascular Surgery, Virginia Commonwealth University Medical Center Medical College of Virginia Campus, West Hospital Building, 7th Floor, South Wing, 1200 East Broad Street, P.O. Box 980068, Richmond, VA 23298-0068, USA
}

This manuscript will review the initial use of CorMatrix in ARE.

\section{Case presentation}

The following seven patients had an ARE procedure performed between October 2011 and April 2013 due to small annular size during AVR:

Case 1 patient. A 79-year-old female who presented with significant shortness of breath, flash pulmonary edema, severe aortic stenosis, ventricular hypertrophy, and diastolic heart failure.

Case 2 patient. A 82-year-old male who presented with shortness of breath, stenosis of the coronary artery (LAD), severe atherosclerosis of the aortic valve, and a thin aortic root.

Case 3 patient. A 40-year-old female who presented with aortic valve murmur and worsening aortic stenosis of her BAV.

Case 4 patient. A 75-year-old male who presented with a history of heart failure, two surgeries involving a coronary artery bypass graft, aortic stenosis, COPD, hypertension, hyperlipidemia, atrial fibrillation, and who had a biventricular ICD.

Case 5 patient. A 61-year-old African-American female who presented with angina and dyspnea with exertion, aortic stenosis, and a history of coronary artery disease and stents.

Case 6 patient. A 44-year-old female who presented with a congenital abnormality in her aortic valve, which was accompanied with severe aortic stenosis. 
Case 7 patient. A 76-year-old female who presented with nonischemic cardiomyopathy, lowered ejection fraction, and who had a biventricular ICD.

ARE was done by utilizing the Nick's procedure to increase the size of the aortic valve implantation. The annular size was increased by extending the aortotomy down to the noncoronary sinus and traversing the annulus down to the anterior leaflet of the mitral valve (Figure 1A,B). The aortic valve prosthesis was then implanted into the enlarged annulus, the patch was then used to augment the aortic root to ensure no impingement on the bioprosthetic, and the defect in the annulus was closed with the CorMatrix ECM patch strut (Figure 1C,D).

All patients had successful aortic root enlargement. The average size of the bioprosthetic valve was $23.3 \pm$ $1.4 \mathrm{~mm}$, and the average mean prosthetic gradient was $12.5 \mathrm{mmHg} \pm 4.3 \mathrm{mmHg}$, the average peak prosthetic gradient was $21.6 \mathrm{mmHg} \pm 6.2 \mathrm{mmHg}$. There were no postoperative reoperations for bleeding, no strokes, and no operative mortality. Average length of stay was 10.3 days \pm 4.1 days. Routine postoperative transthoracic echocardiograms performed revealed normal aortic root appearance.

\section{Conclusions}

There is no definitive conclusion on the best material to use for the reconstruction of cardiac tissue, but an exceptional material would possess tensile strength, hemostatic abilities, calcification resistance, and inability to evoke an immune response [4]. There is evidence that CorMatrix possesses most of these characteristics as it is able to withstand $1200+\mathrm{mmHg}$ of pressure, and has not been demonstrated to undergo calcification nor instigate an immune response [5]. According to the successful outcomes and rapid amelioration of the patients discussed, who displayed no apparent complications with regards to the implanted material, CorMatrix ECM may be better than alternative materials in vivo. The most significant drawbacks of the alternative materials include tearing, dislodgement, erosion, and calcification [4]. Bovine pericardium possesses additional problems including its cellularity which does not promote growth and stimulates an immune response [4]. Based on their compositions, Dacron and polytetrafluoroethylene are unable to promote growth of host tissue as well. CorMatrix ECM is a non-cellular material consisting of only structural proteins, which effectively mimics the natural environment of cells. CorMatrix has been found to readily undergo reabsorption [3] and promotes the growth of a new population of the host's cells [4]. For example, studies of CorMatrix ECM on augmentation cystoplasty showed significant degeneration of the matrix at four weeks post operation, which was accompanied with the proliferation of host ECM and vascularization [3]. By 8 to 12 weeks after the surgery, the implanted matrix was undetectable [3]. A patient who underwent ARE with CorMatrix, displayed normal aortic morphology 30 weeks after the surgery [5]. CorMatrix offers the added benefits of a lower probability of infection and ease of implementation. Although the use of CorMatrix for ARE procedures is promising post-operatively, further studies involving a longer follow up period for the

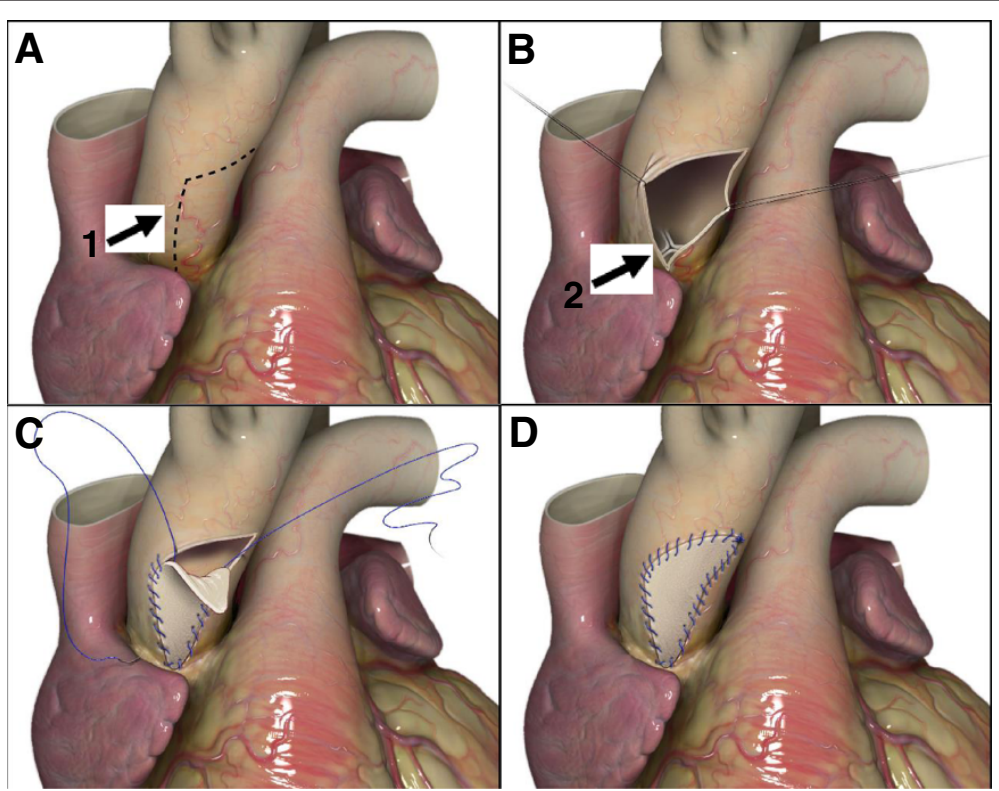

Figure 1 ARE procedure. (A) Incision in the aortic root (1). (B) Opening of the aortic root and exposure of the aortic valve (2). (C) Suturing of CorMatrix patch after AVR. (D) Sealed aortic root. 
patients (i.e. echocardiography) could prove useful and allow for the assessment of longer term efficacy of CorMatrix ECM.

\section{Consent}

Patients in this study have provided written consent to the use of their medical data for academic endeavors, such as submission of a case report to a medical journal, through a consent form that was provided to the patient and/or patient family prior to surgery. A copy of the written consent is available for review by the Editor-inChief of this journal.

\section{Abbreviations}

ARE: Aortic root enlargement; AVR: Aortic valve replacement;

ECM: Extracellular matrix.

\section{Competing interests}

The authors declare that they have no competing interests.

\section{Authors' contributions}

$\mathrm{DB}$ and JP have both contributed to data acquisition, writing of the manuscript and making of the figures. Both authors read and approved the final manuscript.

\section{Acknowledgements}

Virginia Commonwealth University and other staff that have provided assistance to this project.

Received: 2 May 2014 Accepted: 30 October 2014

Published online: 19 November 2014

\section{References}

1. Losenno KL, Gelinas JJ, Johnson M, Chu MW: Defining the efficacy of aortic root enlargement procedures: a comparative analysis of surgical techniques. Can J Cardiol 2013, 29:434-440.

2. Sündermann $\mathrm{SH}$, Biefer HR, Emmert MY, Falk V: Use of extracellular matrix materials in patients with endocarditis. Thorac Cardiovasc Surg 2012, 62(1):76-79.

3. Badylak SF, Kropp B, McPherson T, Liang H, Snyder PW: Small intestinal submucosa: a rapidly resorbed bioscaffold for augmentation cystoplasty in a dog model. Tissue Eng 1998, 4:379-387.

4. Quarti A, Nardone S, Colaneri M, Santoro G, Pozzi M: Preliminary experience in the use of an extracellular matrix to repair congenital heart diseases. Interact Cardiovasc Thorac Surg 2011, 13:569-572.

5. Gerdisch MW, Akinwande AO, Matheny RG: Use of a novel acellular xenograft as a path for aortic annular enlargement during aortic valve replacement. Innovations (Phila) 2010, 5:60-62.

doi:10.1186/s13019-014-0178-5

Cite this article as: Brinster and Patel: The use of CorMatrix extracellular matrix for aortic root enlargement. Journal of Cardiothoracic Surgery 2014 19:178.

\section{Submit your next manuscript to BioMed Central and take full advantage of:}

- Convenient online submission

- Thorough peer review

- No space constraints or color figure charges

- Immediate publication on acceptance

- Inclusion in PubMed, CAS, Scopus and Google Scholar

- Research which is freely available for redistribution 